\title{
Targeting bromodomain protein ANCCA/ATAD2 enhances the efficacy of DNA-damaging chemotherapy agents and radiation
}

\author{
ZHIJIAN DUAN $^{1}$, NICOLAS P. ANDREWS ${ }^{1}$, CHRISTOPHER Z. CHEN $^{2}$, MING FAN $^{2}$, \\ JUNJIAN WANG ${ }^{1,3}$, JING SHEN $^{4}$, JIAN-JIAN LI' ${ }^{2,3}$ and HONG-WU CHEN ${ }^{1,3}$
}

\begin{abstract}
Departments of ${ }^{1}$ Biochemistry and Molecular Medicine,${ }^{2}$ Radiation Oncology and ${ }^{3}$ Comprehensive Cancer Center, University of California, Davis, Sacramento, CA 95817, USA; ${ }^{4}$ Department of Pathology and Pathophysiology, Zhejiang University School of Medicine, Hangzhou, Zhejiang 310058, P.R. China
\end{abstract}

Received April 20, 2019; Accepted October 23, 2019

DOI: 10.3892/or.2019.7418

\begin{abstract}
Bromodomain proteins such as BRD4 chromatin regulator are attractive cancer therapeutic targets. ANCCA (AAA+ nuclear coregulatory cancer-associated protein, also known as ATPase family AAA domain containing 2 or ATAD2) is a novel oncology drug target and contains a bromodomain and an ATPase domain. Our research group as well as others previously identified ANCCA/ATAD2 as a putative oncogene and a poor prognosis factor in many types of cancer including triple-negative breast cancer (TNBC). In the present study, it is reported for the first time that the expression of ANCCA was highly induced by DNA-damaging chemotherapy agents such as carboplatin, doxorubicin and mitomycin $\mathrm{C}$, as well as ionizing radiation. Notably, ANCCA is required for efficient dissolution of DNA damage foci and homologous recombination. Further studies revealed that ANCCA mediates the optimal expression and activation of DNA damage response and repair factors including $\mathrm{Chk} 1, \mathrm{Chk} 2$ and BRCA1, and that ANCCA is recruited to the promoter of BRCA1 in response to DNA damage. Moreover, ANCCA knockdown sensitizes TNBC cells to carboplatin. Collectively, these data provide the first evidence indicating that ANCCA is a novel mediator of DNA damage response and repair and that targeting ANCCA can enhance the efficacy of radiation and chemotherapies.
\end{abstract}

\section{Introduction}

Chromatin regulators play an important role in DNA-templated processes including transcription, DNA replication and repair, and are strongly associated with tumorigenesis (1-3).

Correspondence to: $\mathrm{Dr}$ Hong-Wu Chen, Department of Biochemistry and Molecular Medicine, University of California, Davis, UCDMC Research III Bldg, 4645 2nd Ave, Sacramento, CA 95817, USA

E-mail: hwzchen@ucdavis.edu

Key words: nuclear receptor coactivator, chromatin, epigenetics, gene expression, DNA repair, breast cancer, chemotherapy
We previously identified a bromodomain-containing, AAA+ ATPase protein ANCCA (AAA+ nuclear coregulatory cancer-associated protein, also known as ATPase family AAA domain containing 2 or ATAD2), as a nuclear coactivator for estrogen and androgen receptors $(4,5)$. ANCCA regulates estrogen- or androgen-induced expression of genes involved in proliferation and survival of cancer cells. Further studies demonstrated that ANCCA/ATAD2 is overexpressed in different types of human cancers including breast, lung, liver, gastric, cervical and endometrial cancer, and that its overexpression in TNBC and other cancers is strongly correlated with poor prognosis (6-14). Mechanistically, ANCCA/ATAD2 likely functions by facilitating the assembly of histone-modifying protein complexes at target gene chromatin loci of E2F, Myc and nuclear receptor-regulated genes $(4,5,8,15,16)$. Notably, it was demonstrated that ANCCA/ATAD2 may also be involved in DNA replication through its interactions with acetylated histones (17), indicating that the bromodomain protein may play a role in DNA/chromatin-based processes beyond transcriptional regulation. Recently, a drug discovery campaign by pharmaceutical companies has led to the identification of several small-molecule probes against its bromodomain $(18,19)$, paving the way to development of ANCCA-targeting drugs for treatment of many types of cancer.

DNA damages and consequent aberrant repair are fundamental genetic processes that are often derailed in cell transformation and tumorigenesis (20). On the other hand, anti-cancer radiation and chemotherapy yield clinical benefits by causing genomic instability and DNA damages in cancer cells. Thus, identification of key pathways and regulators for DNA repair in cancer cells, especially those evoked by anti-cancer therapies, can provide important information for enhancing the therapy benefit in cancer treatment. In mammalian cells, there are at least five major mechanisms to repair different types of DNA damage (21). These are base excision repair (BER), mismatch repair (MMR), nucleotide excision repair (NER), and double-strand break repair (DSB), which includes homologous recombination (HR) and non-homologous end joining (NHEJ). Although gene transcription and DNA repair are two seemingly separate processes, accumulating evidence has indicated that they are highly coordinated in response to genotoxic stress conditions (22). For example, 
transcriptional regulation of core factors in BER, NER and MMR facilitates DNA repair, whereas DNA repair factors often monitor the transcription process to maintain fidelity and genome integrity. Recent studies also indicate that transcription factors such as E2F1 can be recruited onto the DNA damage foci where they can directly regulate the DNA repair process (23). One emerging, critical mechanism underlying transcription factors-mediated local DNA repair may be related to their functions to alter chromatin structure and facilitate the repair efficacy (22), suggesting that chromatin regulators with chromatin-remodeling activity could be the extended DNA repair machinery components. In fact, numerous chromatin-modifying proteins and remodeling complexes have been shown to play crucial roles in DNA damage response and repair by facilitating the local chromatin structure change and nucleosome dynamics (24). For example, the recruitment of a lysine methyltransferase (KMT) MMSET/NSD2 to the DSB sites mediates H4K20 methylation which, in turn, facilitates the formation of $\gamma \mathrm{H} 2 \mathrm{AX}-\mathrm{MDC} 1-53 \mathrm{BP} 1$ complex and repair of the damaged DNA (25). Conversely, although bromodomain protein BRD4 in its full-length may promote DNA repair through regulation of the expression of DNA repair genes (26), its shorter isoform can recruit the condensin II chromatin remodeling complex to acetylated histones at the damaged site to inhibit the DNA damage response (27).

In the present study, the possibility that ANCCA is an important responder of cancer cells to genotoxic stress condition was examined. It was revealed that ANCCA protein expression was strongly induced by DNA-damaging, anticancer agents and that increased ANCCA upregulated the expression and activation of key DNA damage response and repair factors including BRCA1. Moreover, it was revealed that ANCCA silencing sensitized TNBC cells to carboplatin. Collectively, these results provide the first evidence indicating that the bromodomain protein ANCCA is an important mediator of DNA damage response and repair in cancer cells and that therapeutics targeting ANCCA hold promise in enhancing chemotherapy efficacy in TNBC.

\section{Materials and methods}

Cell culture, siRNA transfections and drug treatment. MDA-MB-468 and H1299 cells were grown in RPMI-1640 medium or DMEM respectively, supplemented with $10 \%$ fetal bovine serum (Hyclone; GE Healthcare Life Sciences), at $37^{\circ} \mathrm{C}$ in $5 \% \mathrm{CO}_{2}$ incubators. They were purchased from ATCC and recently authenticated using short tandem repeat profiling and used for experiments within 3 to 8 passages after thawing. They were also frequently tested to ensure the absence of mycoplasma. For siRNA transfection, the cells were transfected with Dharmafect 1 (GE Healthcare Dharmacon, Inc.) according to the manufacturer's protocol. The sequences for the siRNAs were ANCCA\#6, GCUACU GUUUACUAUCAGGCU; ANCCA\#7, CAAGCUGCUAAG CCUCCUAUAUU; ANCCA\#58, GUAGGAUUAGAAGUC GUUAUA; ATM, GCGCCUGAUUCGAGAUCCU; ATR, AACCUCCGUGAUGUUGCUUGA; control targeting the luciferase gene, CTTACGCTGAGTACTTCGA. For chemotherapy drug treatments, cells were plated at equal densities, treated with different concentrations of drugs and harvested for western blotting (WB) at different time-points as indicated in the experiments.

$W B$ and $q R T-P C R$. Protein samples were prepared by lysing cells in modified RIPA buffer $(50 \mathrm{mM}$ Tris- $\mathrm{HCl}, \mathrm{pH} 7.5$, $150 \mathrm{mM} \mathrm{NaCl}, 2$ mM EGTA, 5 mM EDTA, $1 \%$ Triton X-100, $0.1 \%$ SDS, $1 \%$ deoxycholate, $10 \mathrm{mM} \mathrm{NaF}, 0.5 \mathrm{mM}$ Na3VO4, and $10 \%$ glycerol). Protein concentrations were measured using Bio-Rad DC Protein Assay kit. Lysates (50-100 $\mu \mathrm{g}$ ) were separated on a $10 \%$ SDS-PAGE gel and transferred to a PVDF membrane. The membrane was then blocked with $5 \%$ milk in $1 \mathrm{X}$ TBST buffer at room temperature for $1 \mathrm{~h}$ with shaking and, after washing in TBST, incubated with different specific primary antibodies overnight at $4^{\circ} \mathrm{C}$. After washing in TBST, the membrane was incubated with appropriate secondary antibody at room temperature for $1 \mathrm{~h}$. Visualization/detection of proteins on the membrane was performed using enhanced chemiluminescence (ECL) WB Reagents from GE Healthcare (RPN2106) followed by exposure to X-ray film. The antibody for ANCCA was generated and purified as previously described (6). Other primary antibodies were obtained from Cell Signaling Technology, Inc. (BRAC1, cat. no. 9010; pBRCA1-S1524, cat. no. 9009; pATR-S428, cat. no. 2853; ATR, cat. no. 2790; pATM-S1981, cat. no. 4526; ATM, cat. no. 2873; pChk1-S345, cat. no. 2348; Chk1, cat. no. 2360; pChk2-T68, cat. no. 2661; and GAPDH, cat. no. 2118) or from Santa Cruz Biotechnology, Inc. (53BP1, cat. no. sc-22760; Rad51, cat. no. sc-8349; E2F1, cat. no. sc-251; $\beta$-actin, cat. no. sc-47778). Antibodies were used at 1:1,000 dilutions, except antibody against beta-actin which was used at 1;2,000 dilutions. qRT-PCR was performed as described with primers reported previously (6).

Immunofluorescence (IF). For IF, after washing with PBS, the cells were fixed by $4 \%$ formaldehyde in PBS for $10 \mathrm{~min}$ at $4^{\circ} \mathrm{C}$. After fixation, the slides were rinsed with PBS. Cells were permeabilized for $10 \mathrm{~min}$ at room temperature (RT) with $0.1 \%$ Triton X-100 in PBS and blocked with 5\% (FBS) in PBS (blocking solution) for $30 \mathrm{~min}$ at RT. After $2 \mathrm{~h}$ of incubation with primary antibodies diluted in the blocking solution and being rinsed three times in PBS, slides were incubated for $1 \mathrm{~h}$ with the appropriate secondary antibodies diluted 1:1,000 in blocking solution. The following antibodies and their dilutions were used: $\gamma \mathrm{H} 2 \mathrm{AX}$ (cat. no. 05-636; 1:200; EMD Millipore), 53BP1 (cat. no. sc-22760; 1:500; Santa Cruz Biotechnology, Inc.), and ANCCA (1:200; homemade described as aforementioned). Following rinses (four times in PBS), slides were mounted with Vectashield mounting medium (Vector Laboratories, Inc.) and sealed with clear nail polish. Images were acquired using a Zeiss LSM510 scanning confocal microscope or Olympus BX61 at the same exposure settings.

Chromatin immunoprecipitation (ChIP) assay. ChIP was performed essentially as previously described (28) with the following modifications. MDA-MB-468 cells were treated with $1 \mu \mathrm{M}$ carboplatin for $12 \mathrm{~h}$ and harvested for ChIP. The crude chromatin solutions were first cleared with Protein A beads (Invitrogen; Thermo Fisher Scientific, Inc.) that were pre-coated with pre-immune serum for $2 \mathrm{~h}$ at $4^{\circ} \mathrm{C}$. The pre-cleared supernatants were then incubated with indicated antibodies at $4^{\circ} \mathrm{C}$ 
overnight prior to precipitation with Protein A beads that had been pre-blocked with BSA and sonicated salmon sperm DNA. ChIP DNA was analyzed by real-time PCR with SYBR-Green (Takara Bio.) on a PCR machine. Enrichment of genomic DNA was presented as the percentage of recovery relative to the input. The primers for BRCA1 promoter were forward, 5'-CGA CTGCTTTGGACAATAGGTAGCG-3' and reverse, 5'-GAG TAGAGGCTAGAGGGCAGGCAC-3'.

Colony formation assay. MDA-MB-468 cells were transfected with indicated siRNAs. Forty-eight hours after transfection, the cells were treated with chemotherapy drugs for $2 \mathrm{~h}$. For MDA-MB-468 cells, $1.0,2.5,5.0$ or $10 \mu \mathrm{M}$ carboplatin were used to obtain a curve. Cells were then trypsinized and plated. After re-plating, the cells were incubated for 10-12 days with medium changed every 3 days. The cells in the plates were then fixed with $10 \%$ formaldehyde in PBS, and stained with $0.5 \%$ crystal violet. The cells were incubated for $30 \mathrm{~min}$ and the stain was removed. The number of cell colonies were counted using ImageJ (version 1.46; National Institutes of Health).

Homologous recombination assay. Homologous recombination (HR) was assessed using a direct repeat green fluorescent protein (DR-GFP) assay as previously described $(29,30)$. Briefly, H1299-DR-GFP cells with an integrated DR-GFP construct were transiently transfected with pCMV3xnlsI-SceI along with different siRNAs separately including control, ANCCA, or RAD51 siRNA. At $72 \mathrm{~h}$ after transfection, the cells were trypsinized and assessed for GFP expression with a BD flow cytometer. The results were analyzed by FlowJo software (version 9.4.9; FlowJo LLC).

Statistical analysis. Results were reported as the means \pm standard deviations. According to the number of groups and variances, data were analyzed with unpaired Student's $t$-test or one-way ANOVA with Tukey's multiple comparison test used for the post hoc test (GraphPad Prism; version 5.0; GraphPad Software, Inc.). Any difference was considered as significant if the probability $<0.05(\mathrm{P}<0.05)$.

\section{Results}

ANCCA protein expression is induced by DNA-damaging drugs and ionizing radiation (IR). Cellular DNA damage response and repair (DDR) involve induction of expression and/or activities of many proteins including the chromatin regulators. Our inspection of ANCCA amino sequences revealed that ANCCA protein contains at its C-terminus, five (S/T)Q sites potentially phosphorylated by ATM/ATR kinases (Fig. 1A). Notably, two of the sites, S1277 and S1302, were demonstrated in a proteomics study to be phosphorylated upon UV-induced DNA damage (31). One common effect of ATM/ATR phosphorylation is to stabilize the substrate protein. To examine whether ANCCA protein level could be altered upon DNA damage, MDA-MB-468 cells, a TNBC cell line, were treated with several DNA-damaging agents and then subjected to immunoblotting analysis. Results revealed in Fig. 1B demonstrated that drugs that cause single and/or double DNA strand breaks, such as cisplatin, carboplatin, doxorubicin (Doxo) and mitomycin C (MMC), induced ANCCA protein expression as early as $6 \mathrm{~h}$ after the drug treatment (Fig. 1B). The induction persisted for at least $24 \mathrm{~h}$. However, paclitaxel (Pacl), which inhibits spindle function, and methotrexate (MTX), which inhibits the metabolism of folic acid and thus the synthesis of macromolecules, did not induce sustained ANCCA expression. MDA-MB-468 cells express a GOF mutant (R273H) form of p53. To examine whether p53 plays a role in the ANCCA induction, a p53-null lung cancer cell line H1299 was treated with the same drugs. Notably, similar results (Fig. 1C) were observed, indicating that cancer cell p53 status is not a crucial factor in the induction. Since IR can cause immediate and direct DNA breaks, H1299 cells were also exposed to IR and ANCCA expression was examined. As early as $2 \mathrm{~h}$ after IR exposure, the induction of ANCCA was detected (Fig. 1D).

It is well known that formation of $\gamma-\mathrm{H} 2 \mathrm{AX}$ (phosphorylated $\mathrm{H} 2 \mathrm{AX}$ ) foci at the DNA damage sites is one of the earliest and most important events in DNA damage response (DDR). To examine whether ANCCA is associated with the DNA damage foci, cells were exposed to IR and stained with $\gamma-\mathrm{H} 2 \mathrm{AX}$ and ANCCA antibodies for confocal IF analysis. The IF analysis revealed that similar to $\gamma$-H2AX, ANCCA could also form punctate structures with an increased intensity after IR treatment (Fig. 1E). However, the majority of ANCCA punctates did not co-localize with $\gamma$-H2AX foci. Only a small fraction of them displayed potential co-localization which were revealed as yellow punctates under the confocal microscope (Fig. 1E). Collectively, the results indicated that, upon DNA damage, ANCCA protein was induced and redistributed in the nucleus of cancer cells.

ANCCA induction is mediated by ATM and ATR. ATM and ATR kinases are central upstream regulators of DDR. To investigate whether DNA damage-induced ANCCA expression is regulated by the ATM/ATR pathway, ATM or ATR were knocked down before treatment with drugs. Results in Fig. 2A revealed that in comparison to siControl, silencing of ATM in MDA-MB-468 cells markedly decreased ANCCA induction by carboplatin or doxorubicin. Likewise, in comparison to the siControl, knockdown of ATR also effectively mitigated the induction (Fig. 2A). However, in regularly growing cells without drug treatment, ATM or ATR knockdown did not affect ANCCA expression in the Western blotting. Furthermore, treatment of the cells with caffeine (a general ATM and ATR inhibitor) or KU60019 (a specific inhibitor of ATM) almost completely eliminated carboplatin or doxorubicin-induced ANCCA upregulation (Fig. 2B). These results strongly indicated that DNA-damaging drug-induced ANCCA protein induction was mediated by the key DDR regulators ATM and ATR.

ANCCA silencing does not affect DNA damage foci formation but delays their dissolution. It was next examined whether ANCCA silencing influenced $\gamma$-H2AX foci formation or their dissolution. The dissolution occurs during or after the completion of the repair. Immunofluorescence analysis revealed that $\gamma$-H2AX foci were rapidly generated $2 \mathrm{~h}$ after radiation in both the ANCCA-silenced group and the control group (Fig. 3A and B), indicating that ANCCA does not play a critical role in the formation of $\gamma-\mathrm{H} 2 \mathrm{AX}$ 


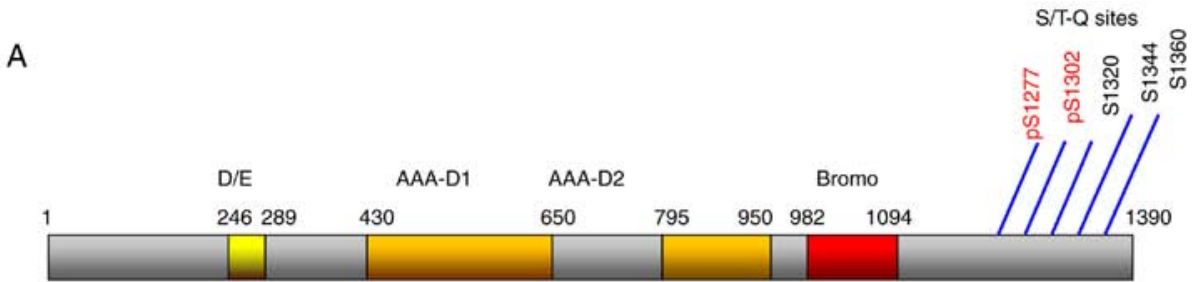

B

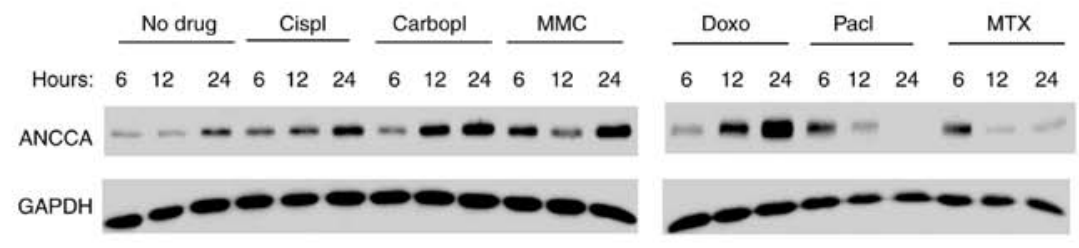

C

D
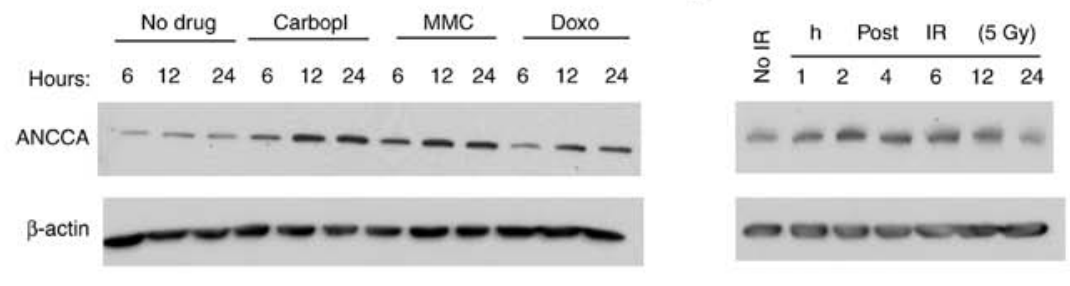

$\mathrm{E}$
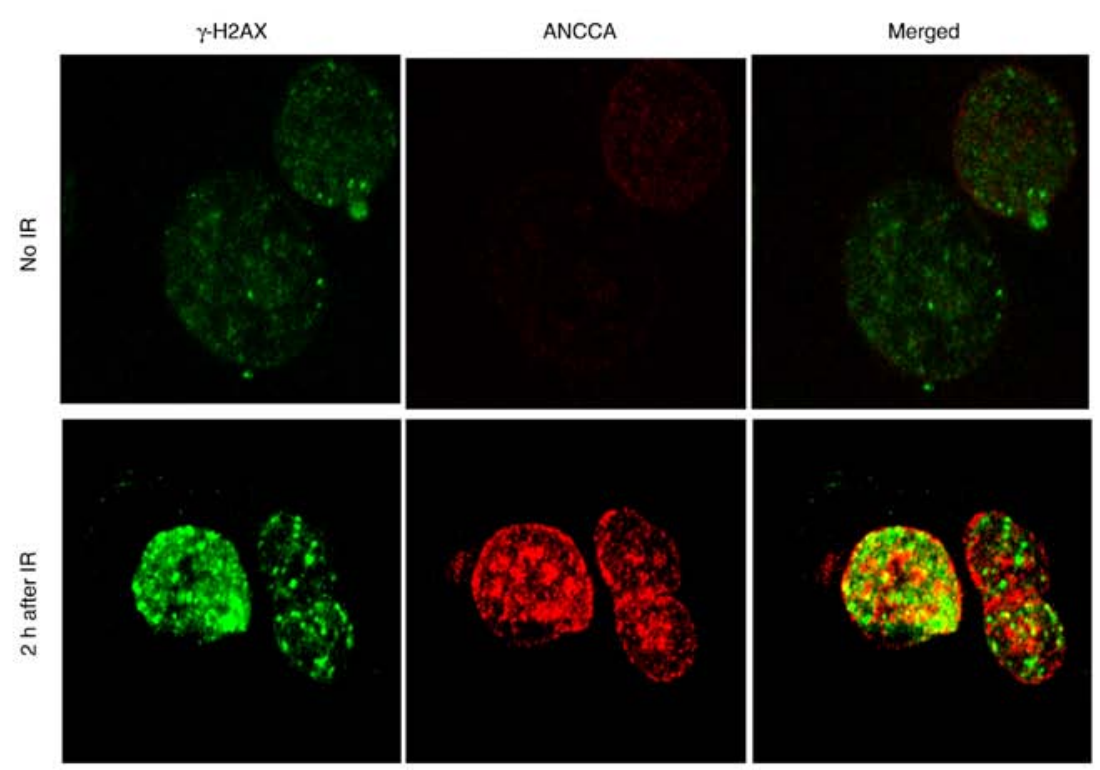

Figure 1. ANCCA is induced in cancer cells upon DNA damage. (A) Schematic diagram of ANCCA/ATAD2 protein with indicated functional domains and potential ATM or ATR phosphorylation sites. (B) MDA-MB-468 cells were treated with $10 \mu \mathrm{M}$ Cispl, $20 \mu \mathrm{M}$ Carbopl, $1.0 \mu \mathrm{M}$ MMC, $0.1 \mu \mathrm{M}$ Doxo, $0.1 \mu \mathrm{M}$ Pacl, 1.0 $\mu \mathrm{M}$ MTX or vehicle for indicated time-points. Cells were then harvested for immunoblotting. (C) H1299 cells were treated with the indicated chemo-drugs as in A before being harvested for immunoblotting. (D) H1299 cells were exposed to IR at a dose of 5 Gy and harvested at indicated time-points after IR before being harvested for immunoblotting. (E) H1299 cells were exposed to IR at a dose of $5 \mathrm{~Gy}$ for $2 \mathrm{~h}$ before being harvested for immune-staining with rH2A.x (Alexa 488-labeled) or ANCCA antibody. ANCCA, AAA+ nuclear coregulatory cancer-associated protein; Cispl, cisplatin; Carbopl, carboplatin; MMC, mitomycin C; Doxo, doxorubincin; Pacl, paclitaxel; MTX, methotrexate; IR, ionizing radiation.

foci. Notably, when the dissolution of $\gamma$-H2AX foci was analyzed, a significant delay in the ANCCA-silenced group was detected, with foci remaining $\sim 2$-fold higher than the control group at $24 \mathrm{~h}$ after IR. In addition, the foci formation of another important DDR protein 53BP1 was also analyzed following exposure to DNA-damaging agent MMC. At $48 \mathrm{~h}$ after MMC treatment, the ANCCA-silenced group still had a higher percentage of 53BP1-positive cells than the control group (data not shown). These results indicated that although ANCCA may not play an essential role in the initial step of DDR, it may be involved in the timely completion of the repair process of DNA damage.

ANCCA controls CHK1 and CHK2 signaling and the expression of BRCA1. To explore the possible function of ANCCA in DNA damage response and repair, ANCCA was silenced by siRNA and the effect on signaling and the expression of major DDR proteins was examined. As revealed in Fig. 4A, consistent with previous studies, treatment of cells with the genotoxic drugs (i.e., MMC, carboplatin and doxorubicin) 


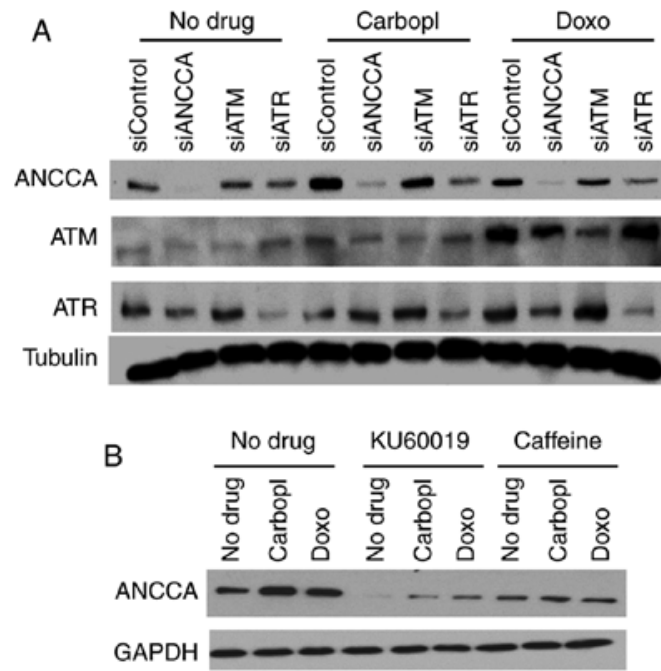

Figure 2. ANCCA protein level is regulated by ATM/ATR pathway. (A) MDA-MB-468 cells transfected with siRNAs for $48 \mathrm{~h}$ were treated with $20 \mu \mathrm{M}$ Carbopl or $0.1 \mu \mathrm{M}$ Doxo for $12 \mathrm{~h}$ before being harvested for immunoblotting. (B) MDA-MB-468 cells were treated with $20 \mu \mathrm{M}$ Carbopl, $0.1 \mu \mathrm{M}$ Doxo or vehicle, in combination with $10 \mu \mathrm{M}$ KU60019 or $4 \mathrm{mM}$ caffeine for $12 \mathrm{~h}$ before being harvested for immunoblotting. ANCCA, AAA+ nuclear coregulatory cancer-associated protein; Carbopl, carboplatin; Doxo, doxorubincin.

induced a DNA damage response with strong activation of CHK1 and CHK2 kinases as indicated by their markedly increased phosphorylation and the increased phosphorylation of the BRCA1 protein. Notably, ANCCA depletion strongly diminished the activation of Chk1 and Chk2 as well as the activation of BRCA1. Similar effects were also observed in cells treated with IR (Fig. 4B). Notably, ANCCA silencing also decreased the total protein level of BRCA1 elevated by carboplatin and doxorubicin or IR (Fig. 4A and B). It has been revealed that effective DNA damage response and repair necessitates transcriptional upregulation of genes involved in DDR including BRCA1 $(32,33)$. Consistent with previous studies, carboplatin and doxorubicin treatment significantly increased BRCA mRNA expression. However, the increase was largely diminished by ANCCA knockdown (Fig. 4C). Since ANCCA is a chromatin-bound protein and has been revealed to function as a coactivator of transcription factors such as ER, AR or E2F1, it was further investigated whether ANCCA could regulate BRCA1 gene expression directly. In fact, ChIP analysis revealed that ANCCA was recruited to BRCA1 gene promoter, and that carboplatin treatment significantly enhanced the ANCCA recruitment (Fig. 4D). Notably, the treatment also increased the accumulation of transcriptionally active histone mark $\mathrm{H} 3 \mathrm{~K} 4 \mathrm{me} 3$ and the H3K4 methyltransferase MLL at BRCA1 promoter (Fig. 4D), indicating that ANCCA may mediate the chemotherapy drug induction of BRCA1 expression through recruitment of MLL1 and H3K4 methylation of local chromatin.

ANCCA knockdown affects homologous recombination. BRCA1 is important in homologous recombination (HR), one of the major pathways for DNA repair and cell survival. Having determined that ANCCA controls the expression and activation of BRAC1, it was next examined whether ANCCA plays a
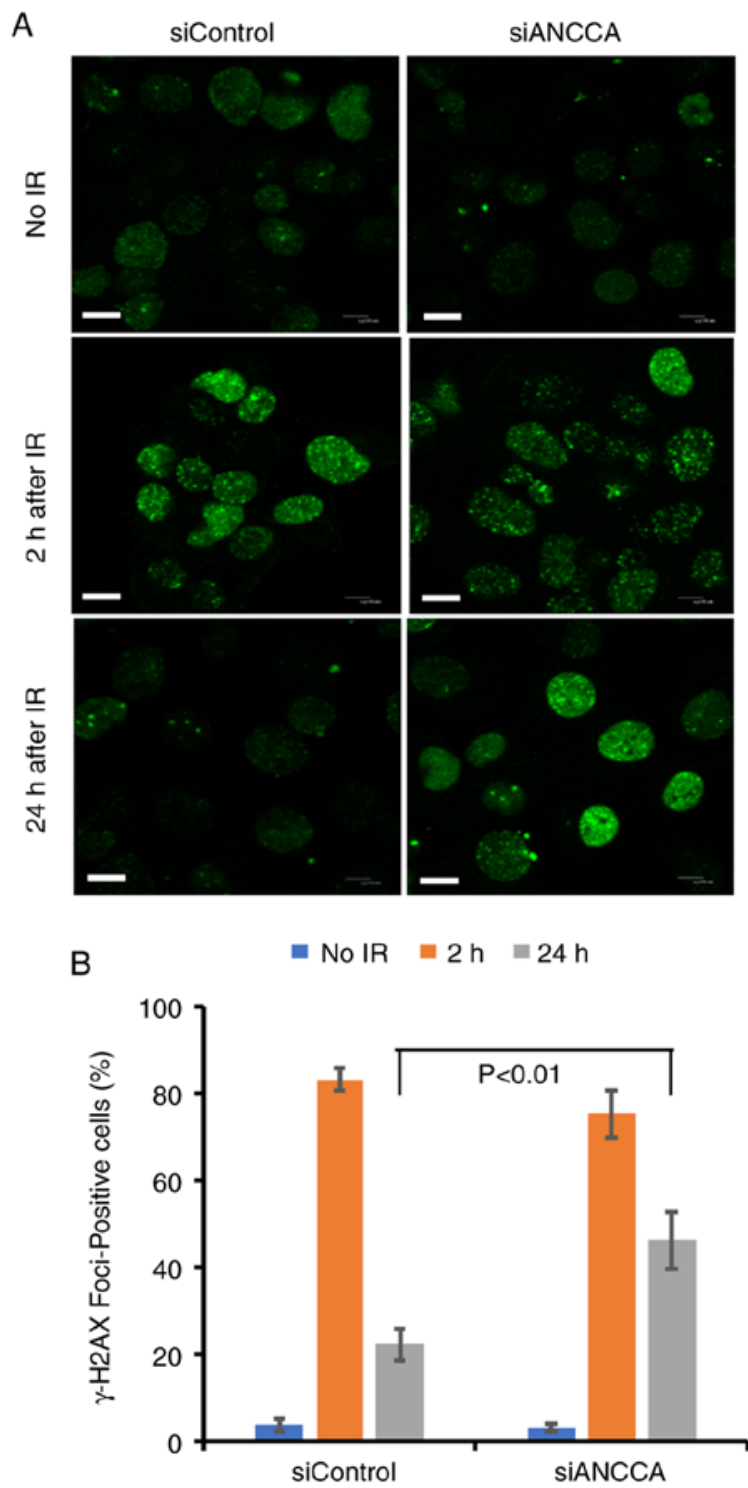

Figure 3. ANCCA silencing does not affect DNA damage foci formation but delays their dissolution. H1299 cells transfected with siRNAs for $48 \mathrm{~h}$ were exposed to ionizing radiation at a dose of $5 \mathrm{~Gy}$. Cells were harvested at 2 and $24 \mathrm{~h}$ later and stained with $\gamma$-H2AX (Alexa 488-labeled). (A) Representative images were captured under a confocal microscope. Scale bars, $10 \mu \mathrm{m}$. (B) Cells with 5 or more foci were considered $\gamma$-H2AX foci-positive cells. Under the microscope, 500 cells were counted and analyzed. Data presented are the mean \pm SD from three independent experiments. $\mathrm{P}<0.01$. ANCCA, $\mathrm{AAA}+$ nuclear coregulatory cancer-associated protein.

role in modulating HR. A DR-GFP assay was thus performed. H1299-DR-GFP cells were co-transfected with I-SceI endonuclease expression plasmid and either control siRNA, ANCCA siRNA or Rad51 siRNA. Cells were harvested at $72 \mathrm{~h}$ after transfection to allow sufficient recombination to occur. Similar to the HR inhibition effect (to $22 \%$ of control) by depletion of Rad51, the key component of HR, knockdown of ANCCA also significantly reduced HR repair efficiency to $\sim 43$ to $60 \%$ by the different siRNAs, indicating an important role of ANCCA in the HR repair pathway (Fig. 5).

ANCCA knockdown sensitizes TNBC cells to DNA-damaging drugs. Given the important functions of ANCCA in DNA damage response and repair, whether ANCCA silencing 

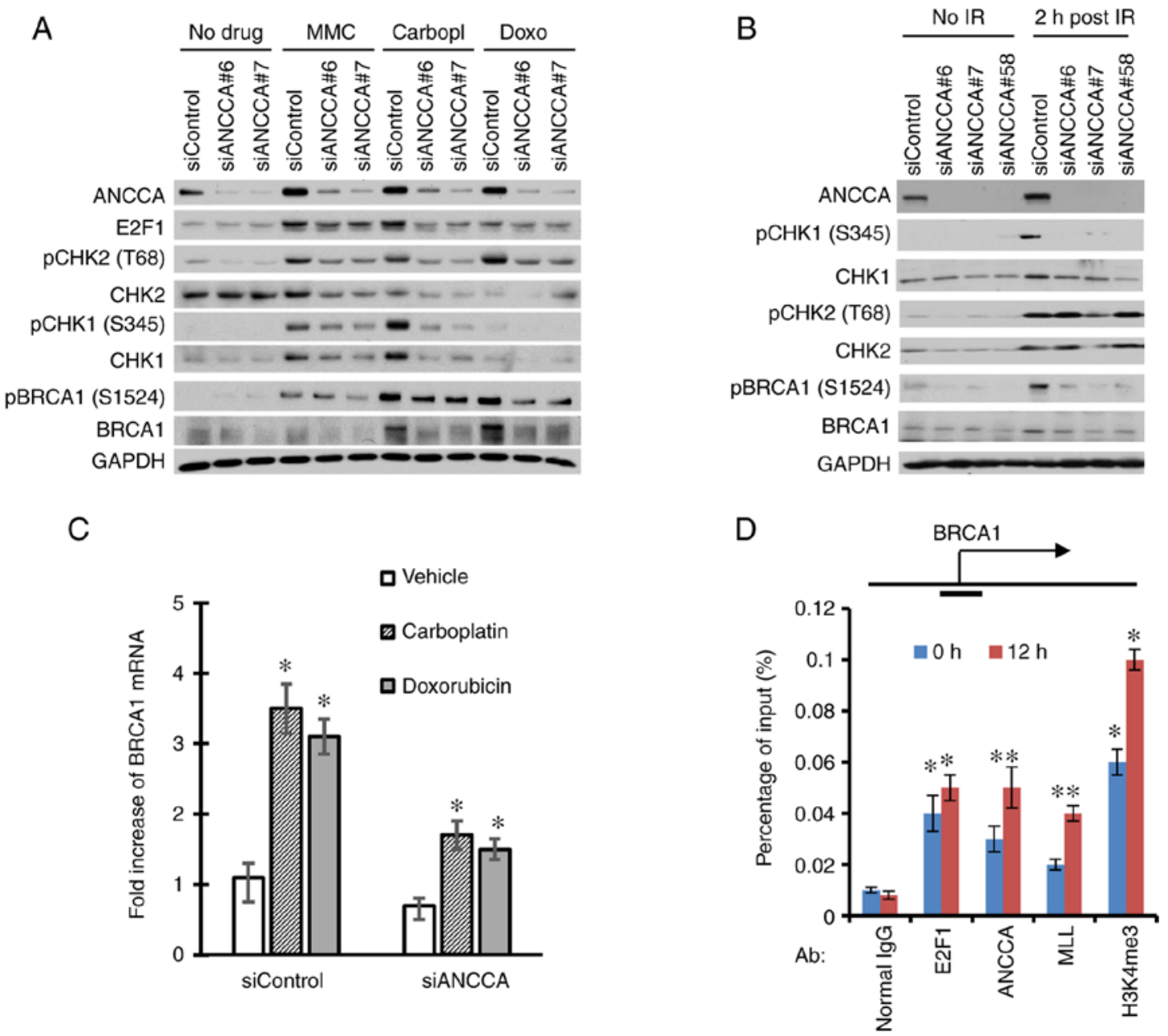

Figure 4. ANCCA silencing suppresses the expression of DNA damage response and repair proteins. (A) MDA-MB-468 cells transfected with siRNAs for ANCCA or control were treated with $20 \mu \mathrm{M}$ Carbopl, $1.0 \mu \mathrm{M}$ MMC, $0.1 \mu \mathrm{M}$ Doxo or without drugs for $12 \mathrm{~h}$ before being harvested for immunoblotting with indicated antibodies. (B) H1299 cells transfected with the siRNAs were exposed to at a dose of $5 \mathrm{~Gy}$ for $2 \mathrm{~h}$ before being harvested for immunoblotting. (C) MDA-MB-468 cells transfected with siRNAs for $48 \mathrm{~h}$ were treated with $20 \mu \mathrm{M}$ Carbopl, $0.1 \mu \mathrm{M}$ Doxo or vehicle for $24 \mathrm{~h}$ before being harvested for RT-PCR analysis of BRCA1 expression. "P<0.002. (D) MDA-MB-468 cells treated with $20 \mu \mathrm{M}$ Carbopl for $12 \mathrm{~h}$ were harvested for ChIP with indicated antibodies. Relative occupancy of indicated proteins at the BRCA1 gene was analyzed by qPCR of ChIP DNA with primers amplifying the promoter region indicated by a line. Data are presented as the mean $\pm \mathrm{SD}$ from three independent experiments. $\mathrm{P}<0.001$ and ${ }^{* *} \mathrm{P}<0.01$ when compared to IgG control. ANCCA, $\mathrm{AAA}+$ nuclear coregulatory cancer-associated protein; Carbopl, carboplatin; MMC, mitomycin C; Doxo, doxorubincin; IR, ionizing radiation.

affects the efficacy of DNA-damaging drugs was next examined. Thus, colony formation assays were performed with MDA-MB-468 TNBC cells treated by a combination of ANCCA siRNA and carboplatin. Results in Fig. 6 revealed that when compared to the control siRNA, ANCCA knockdown alone markedly decreased the number of colonies (from 323 in the siControl to 251 in siANCCA\#6), which is consistent with our previous findings that ANCCA plays a positive role in promoting breast cancer cell proliferation and survival (6). Notably, when cells were also treated with different concentrations of carboplatin, more marked decreases of colony numbers in cells with ANCCA knockdown were observed than in cells with siRNA-control. For instance, at $1 \mu \mathrm{M}$ carboplatin, colonies of si-ANCCA- 6 cells decreased $\sim 45 \%$ (from 251 in vehicle-treated to 139 in $1 \mu \mathrm{M}$ carboplatin-treated) whereas colonies of siRNA-control cells decreased only $\sim 10 \%$ (from 323 to 291). Likewise, $2.5 \mu \mathrm{M}$ carboplatin treatment of siANCCA\#6 cells caused $>75 \%$ reduction in colony numbers whereas it took $10 \mu \mathrm{M}$ of the drug to cause a similar effect. Therefore, ANCCA silencing could strongly sensitize cancer cells to killing by DNA-damaging drugs such as carboplatin.

\section{Discussion}

Genotoxic stress or DNA damage elicits cellular DDR responses that are complex and integrated to ensure the survival of both normal and cancer cells. In the context of cancer cells, identification of major cellular DDR factors can be of value in providing new strategies in enhancing the efficacy of chemotherapies. In the present study, several lines of evidence were provided that ANCCA, which has a bromodomain that associates with acetylated histones and functions in transcriptional regulation $(7,15)$, is also involved in DNA damage repair. First, it was revealed that ANCCA protein was strongly induced in response to various DNA-damaging agents, including chemotherapeutic drugs and IR, which requires the activity of DNA damage-activated kinases (ATM and ATR). Secondly, it was demonstrated that ANCCA knockdown significantly delayed $\gamma-\mathrm{H} 2 \mathrm{AX}$ and 53BP1 foci dissolution. Thirdly, it was revealed 

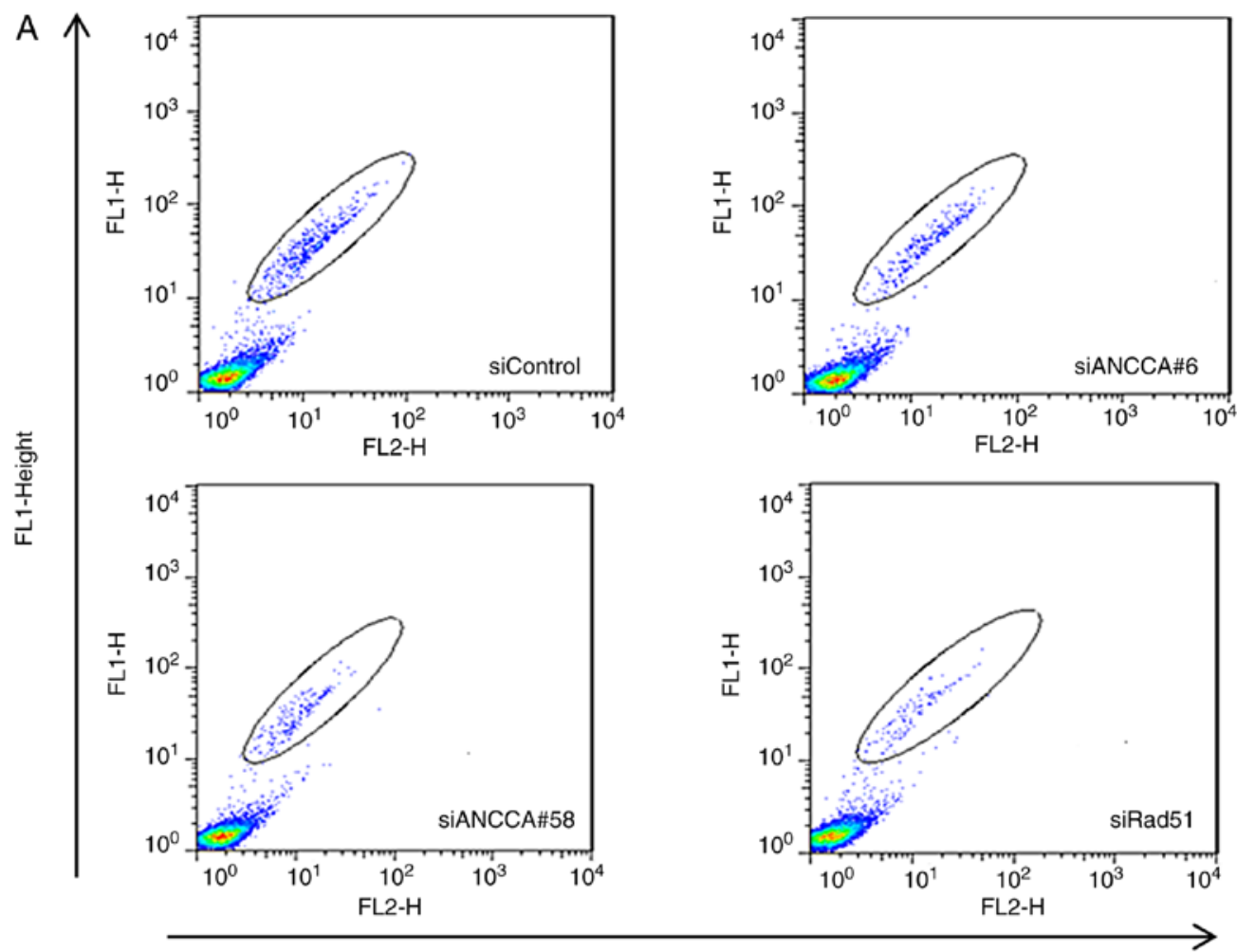

FL2-Height

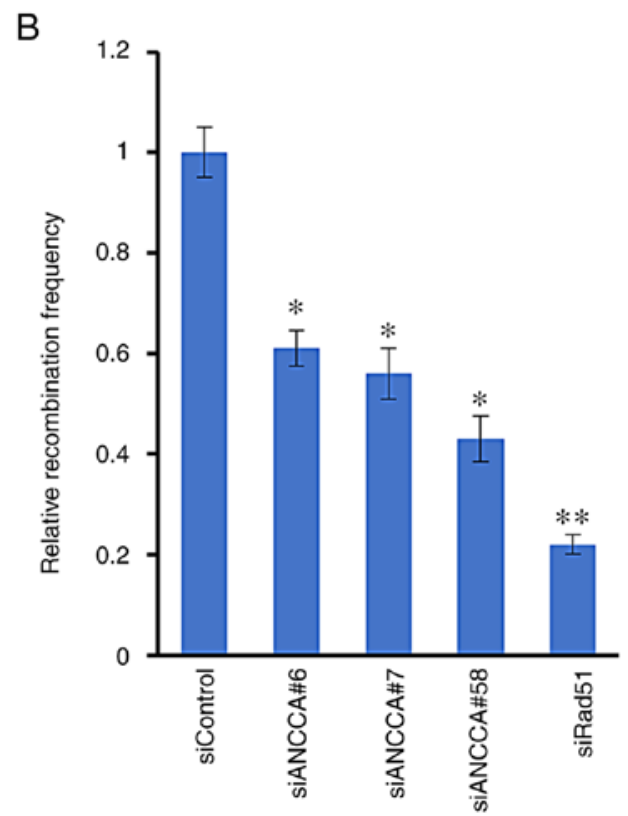

Figure 5. ANCCA knockdown affects HR. (A) HR efficiency-reporting H1299-DR-GFP cells were transfected with an endonuclease expressing plasmid together with siRNAs against ANCCA, RAD51 or control sequence. Cells were then assessed for GFP expression by flow cytometry. (A) Representative dot plots of flow analysis of $\mathrm{GFP}^{+}$cells. Cells that have undergone HR are circled. (B) ANCCA or RAD51 knockdown reduced the HR efficiency. Data presented are the mean of three independent experiments. ${ }^{*} \mathrm{P}<0.01$ and ${ }^{* * *} \mathrm{P}<0.001$. ANCCA, AAA+ nuclear coregulatory cancer-associated protein; HR, homologous recombination; GFP, green fluorescent protein.

that ANCCA depletion inhibited the expression and activation of numerous DNA damage response factors, including Chk1, Chk2 and BRCA1. Finally, it was demonstrated that ANCCA knockdown also severely impaired the HR-dependent repair efficiency.

Unlike many DDR factors, ANCCA is abundantly expressed in cells and tumors of many types of cancers. Yet, it was revealed that its protein level was strongly induced by
DNA damage agents and that this induction could be readily observed within $2 \mathrm{~h}$ of IR or $6 \mathrm{~h}$ of chemo-drug treatment. Further analysis indicated that the ANCCA induction does not appear to involve transcriptional activation (data not shown). Multiple putative ATM/ATR-mediated phosphorylation sites can be identified at the C-terminus of ANCCA. A previous proteomics study revealed that at least two of the putative ATM/ATR phosphorylation sites at the C-terminus of ANCCA 
A
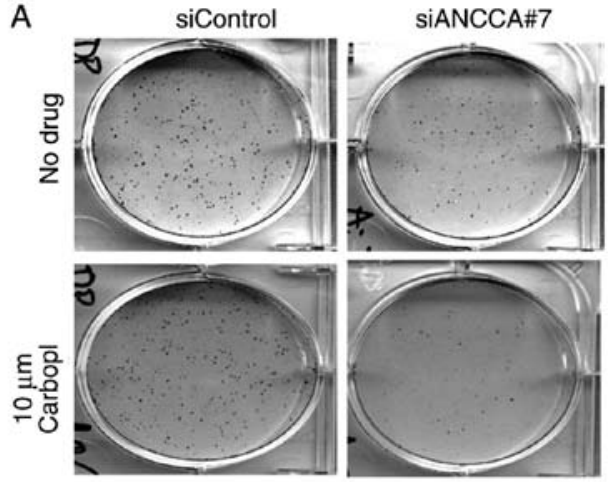

B

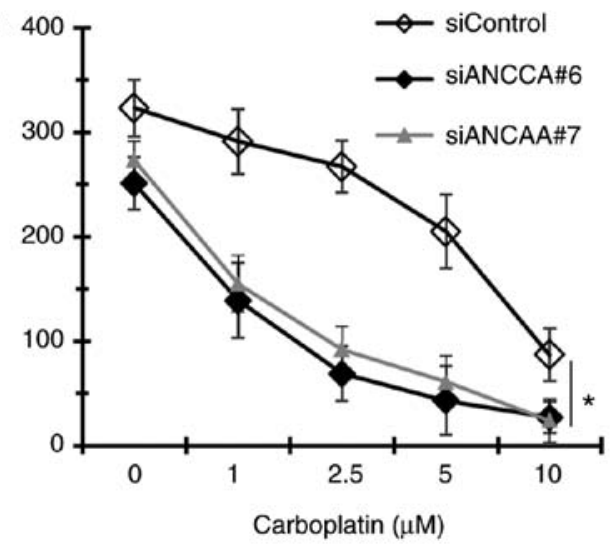

Figure 6. ANCCA knockdown sensitizes TNBC cells to carboplatin. (A) MDA-MB-468 cells were transfected with indicated siRNAs. Forty-eight hours after transfection, cells were treated with carboplatin for $2 \mathrm{~h}$ and then plated to new 6-well plates and incubated for 10-12 days before staining with crystal violet for colony counting. Representative images of colony formation by the indicated treatments are presented. (B) The number of cell colonies were counted using ImageJ. Data presented are the mean \pm SD from three independent experiments. "P $<0.01$. Student's t-test. ANCCA, AAA+ nuclear coregulatory cancer-associated protein; $\mathrm{TNBC}$, triple-negative breast cancer.

(S1277 and S1302) could be phosphorylated by UV-induced DNA damage (31). Although the exact role of the phosphorylation events is unclear, it is tempting to speculate that the phosphorylation at either or both of the sites by ATM/ATR in response to genotoxic stress upregulates the stability of ANCCA protein. In support of this hypothesis, it was revealed that suppression of the expression or function of ATM and ATR effectively diminished ANCCA induction.

The exact role and functional mechanisms of ANCCA involvement in DDR are unclear at this point. One important role revealed in this study is its direct control of BRCA1. As one of the core factors of $\mathrm{HR}, \mathrm{BRCA} 1$ expression regulation in response to DNA damage has been revealed to be dynamic and sometimes context-dependent $(32,33)$. Several transcriptional activating and repressing complexes containing factors such as $\mathrm{E} 2 \mathrm{~F} 1, \mathrm{Rb}$ and BRCA1 itself were revealed to be important in control of BRCA1 gene transcription. Effective assembly and disassembly of the complexes at BRCA1 gene regulatory enhancer and promoter will likely be part of the important regulatory events that underlie the dynamics of its regulation in response to DNA damage. Given the AAA type of ATPase possessed by ANCCA, it can be envisaged that through its physical association with acetylated histones via its bromodomain and with DNA-binding factors such as E2Fs,
ANCCA can play a pivotal role in facilitating the assembly and/or disassembly of the regulatory complexes. The present ChIP data lend some support to this model. Notably, a recent study demonstrated that overexpressed ATAD2/ANCCA could increase the expression of PLK4, a serine/threonine kinase, which plays important functions in tumorigenesis and radiation resistance in glioblastoma models (34), which is in line with the notion that ANCCA is a major mediator of DNA damage-based therapeutic resistance. To date, the exact mechanism(s) of how ANCCA depletion led to the diminished activation of Chk1 and Chk2 is unclear. One possibility is that ANCCA depletion causes decreased expression of BRCA1, which in turn leads to diminished Chk1 activation. As well established, BRCA1 can regulate the expression and activation of Chk1. Alternatively, ANCCA depletion inhibited ATM protein expression as revealed in Fig. 2A, which in turn diminished Chk1 and Chk2 activation.

In addition to the transcriptional regulation of DNA repair genes, it was also observed that ANCCA depletion not only reduced the expression of Chk1 and Chk2 and BRCA1 but also their phosphorylation (and hence activation). These results raise the possibility that DNA damage-induced ANCCA may also participate in some of the DNA damage signaling and/or repair processes. One major feature of genotoxic insults is a change of histone acetylation landscape at the damaged chromatin (26). One recent proteomics study revealed that, in addition to proteins of chromatin remodeling complexes as anticipated, ANCCA is also associated with proteins and enzymes of DNA replication and repair (35). The latter includes Top2a, PARP1, and BLM. Considering that ANCCA has a bromodomain that associates with the acetylated $\mathrm{H} 3$ and $\mathrm{H} 4$, it is thus possible that enhanced histone acetylations at the damaged loci guide ANCCA recruitment. Once recruited, ANCCA can assist the repair by facilitating assembly and/or loading of repair complexes through its physical association with the complexes and its ATPase activity. This hypothetical recruitment mode is consistent with our IF data in Fig. 1E and our failure to detect ANCCA association with $\gamma$-H2AX in co-IP experiments (data not shown). Future studies are required to examine the model with small-molecule inhibitors specifically targeting ANCCA bromodomain or its ATPase, once the inhibitors become available for use in cell cultures or animal models. Further studies with the inhibitors can also be conducted to examine whether targeting ANCCA in combination with different chemotherapeutics (e.g., Topo-II inhibitors such as doxorubicin and DNA crosslinking agents such as carboplatin) can elicit different therapeutic efficacy.

Our previous studies demonstrated that ANCCA overexpression may serve as a poor prognostic marker for TNBC and that it is crucial for proliferation and survival of cancer cells (6). In addition, ANCCA was also revealed to control the expression of B-Myb, histone methyltransferase EZH2 and an $\mathrm{Rb}-\mathrm{E} 2 \mathrm{~F}$ core program for proliferation, as well as a subset of mitotic kinesins and survival genes $(6,15,36)$. Collectively with our findings of the functions of ANCCA in DNA damage repair for TNBC cells, our studies revealed that ANCCA can be a valuable target for the treatment of TNBC. In particular, inhibition of ANCCA may resensitize tumors of advanced TNBC to chemotherapy or radiation. Moreover, findings of this study also provide rationale for determination of whether 
ANCCA overexpression represents a prognostic factor for early relapse from certain chemotherapies or radiation therapy.

\section{Acknowledgements}

We would like to thank Dr R.G Bristow for H1299-DR-GFP cells and Mr. Neeraj Lal for technical help.

\section{Funding}

The present study was supported by the NIH grants R01CA113860 and R01CA224900 (HWC) and R01CA213830 (JJL). NPA was a trainee of an NIH T32 training grant.

\section{Availability of data and materials}

The materials used in this study are available from the corresponding author upon reasonable request. All data analyzed in this study are included in this article.

\section{Authors' contributions}

ZD, NPA, JJL and HWC designed the experiments. ZD, NPA, CZC, MF, JW and JS performed the experiments and analyzed the data. ZD, CZC, JS, JJL and HWC wrote the manuscript. JJL and HWC edited the manuscript. All authors read and approved the final manuscript and agree to be accountable for all aspects of the research in ensuring that the accuracy or integrity of any part of the work are appropriately investigated and resolved.

\section{Ethics approval and consent to participate}

Not applicable.

\section{Patient consent for publication}

Not applicable.

\section{Competing interests}

The authors declare that they have no competing interests.

\section{References}

1. Liu B, Yip R and Zhou Z: Chromatin remodeling, DNA damage repair and aging. Curr Genomics 13: 533-547, 2012.

2. Soria G, Polo SE and Almouzni G: Prime, repair, restore: The active role of chromatin in the DNA damage response. Mol Cell 46: 722-734, 2012

3. Price BD and D'Andrea AD: Chromatin remodeling at DNA double-strand breaks. Cell 152: 1344-1354, 2013.

4. Zou JX, Guo L, Revenko AS, Tepper CG, Gemo AT, Kung HJ and Chen HW: Androgen-induced coactivator ANCCA mediates specific androgen receptor signaling in prostate cancer. Cancer Res 69: 3339-3346, 2009.

5. Zou JX, Revenko AS, Li LB, Gemo AT and Chen HW: ANCCA, an estrogen-regulated AAA+ ATPase coactivator for ERalpha, is required for coregulator occupancy and chromatin modification. Proc Natl Acad Sci USA 104: 18067-18072, 2007.

6. Kalashnikova EV, Revenko AS, Gemo AT, Andrews NP, Tepper CG, Zou JX, Cardiff RD, Borowsky AD and Chen HW: ANCCA/ATAD2 overexpression identifies breast cancer patients with poor prognosis, acting to drive proliferation and survival of triple-negative cells through control of B-Myb and EZH2. Cancer Res 70: 9402-9412, 2010.
7. Caron C, Lestrat C, Marsal S, Escoffier E, Curtet S, Virolle V, Barbry P, Debernardi A, Brambilla C, Brambilla E, et al: Functional characterization of ATAD2 as a new cancer/testis factor and a predictor of poor prognosis in breast and lung cancers. Oncogene 29: 5171-5181, 2010.

8. Ciro M, Prosperini E, Quarto M, Grazini U, Walfridsson J, McBlane F, Nucifero P, Pacchiana G, Capra M, Christensen J and Helin K: ATAD2 is a novel cofactor for MYC, overexpressed and amplified in aggressive tumors. Cancer Res 69: 8491-8498, 2009.

9. Zhang M, Zhang C, Du W, Yang X and Chen Z: ATAD2 is overexpressed in gastric cancer and serves as an independent poor prognostic biomarker. Clin Transl Oncol 18: 776-781, 2016.

10. Yang J, Huang J, Luo L, Chen Z, Guo Y and Guo L: Significance of PRO2000/ANCCA expression, a novel proliferation-associated protein in hepatocellular carcinoma. Cancer Cell Int 14: 33, 2014.

11. Krakstad C, Tangen IL, Hoivik EA, Halle MK, Berg A, Werner HM, Ræder MB, Kusonmano K, Zou JX, Øyan AM, et al: ATAD2 overexpression links to enrichment of B-MYB-translational signatures and development of aggressive endometrial carcinoma. Oncotarget 6: 28440-28452, 2015.

12. Hwang HW, Ha SY, Bang H and Park CK: ATAD2 as a poor prognostic marker for hepatocellular carcinoma after curative resection. Cancer Res Treat 47: 853-861, 2015.

13. Shang P, Meng F, Liu Y and Chen X: Overexpression of ANCCA/ATAD2 in endometrial carcinoma and its correlation with tumor progression and poor prognosis. Tumour Biol 36: 4479-4485, 2015 .

14. Zheng L, Li T, Zhang Y, Guo Y, Yao J, Dou L and Guo K: Oncogene ATAD2 promotes cell proliferation, invasion and migration in cervical cancer. Oncol Rep 33: 2337-2344, 2015.

15. Revenko AS, Kalashnikova EV, Gemo AT, Zou JX and Chen HW: Chromatin loading of E2F-MLL complex by cancer-associated coregulator ANCCA via reading a specific histone mark. Mol Cell Biol 30: 5260-5272, 2010.

16. Huang J, Yang J, Lei Y, Gao H, Wei T, Luo L, Zhang F, Chen H, Zeng Q and Guo L: An ANCCA/PRO2000-miR-520a-E2F2 regulatory loop as a driving force for the development of hepatocellular carcinoma. Oncogenesis 5: e229, 2016.

17. Koo SJ, Fernández-Montalván AE, Badock V, Ott CJ, Holton SJ, von Ahsen O, Toedling J, Vittori S, Bradner JE and Gorjánácz M: ATAD2 is an epigenetic reader of newly synthesized histone marks during DNA replication. Oncotarget 7: 70323-70335, 2016.

18. Bamborough P, Chung CW, Furze RC, Grandi P, Michon AM, Watson RJ, Mitchell DJ, Barnett H, Prinjha RK, Rau C, et al: Aiming to miss a moving target: Bromo and extra terminal domain (BET) selectivity in constrained ATAD2 inhibitors. J Med Chem 61: 8321-8336, 2018.

19. Fernández-Montalván AE, Berger M, Kuropka B, Koo SJ, Badock V, Weiske J, Puetter V, Holton SJ, Stöckigt D, Ter Laak A, et al: Isoform-selective ATAD2 chemical probe with novel chemical structure and unusual mode of action. ACS Chem Biol 12: 2730-2736, 2017.

20. Lord CJ and Ashworth A: The DNA damage response and cancer therapy. Nature 481: 287-294, 2012.

21. Altieri F, Grillo C, Maceroni M and Chichiarelli S: DNA damage and repair: From molecular mechanisms to health implications. Antioxid Redox Signal 10: 891-937, 2008.

22. Fong YW, Cattoglio $\mathrm{C}$ and Tjian R: The intertwined roles of transcription and repair proteins. Mol Cell 52: 291-302, 2013.

23. Biswas AK and Johnson DG: Transcriptional and nontranscriptional functions of E2F1 in response to DNA damage. Cancer Res 72: 13-17, 2012.

24. Hauer MH and Gasser SM: Chromatin and nucleosome dynamics in DNA damage and repair. Genes Dev 31: 2204-2221, 2017.

25. Pei H, Zhang L, Luo K, Qin Y, Chesi M, Fei F, Bergsagel PL, Wang L, You Z and Lou Z: MMSET regulates histone H4K20 methylation and 53BP1 accumulation at DNA damage sites. Nature 470: 124-128, 2011.

26. Li X, Baek G, Ramanand SG, Sharp A, Gao Y, Yuan W, Welti J, Rodrigues DN, Dolling D, Figueiredo I, et al: BRD4 promotes DNA repair and mediates the formation of TMPRSS2-ERG gene rearrangements in prostate cancer. Cell Rep 22: 796-808, 2018.

27. Floyd SR, Pacold ME, Huang Q, Clarke SM, Lam FC, Cannell IG, Bryson BD, Rameseder J, Lee MJ, Blake EJ, et al: The bromodomain protein Brd4 insulates chromatin from DNA damage signalling. Nature 498: 246-250, 2013.

28. Wang J, Zou JX, Xue X, Cai D, Zhang Y, Duan Z, Xiang Q, Yang JC, Louie MC, Borowsky AD, et al: ROR- $\gamma$ drives androgen receptor expression and represents a therapeutic target in castration-resistant prostate cancer. Nat Med 22: 488-496, 2016. 
29. Chan N, Koritzinsky M, Zhao H, Bindra R, Glazer PM, Powell S, Belmaaza A, Wouters B and Bristow RG: Chronic hypoxia decreases synthesis of homologous recombination proteins to offset chemoresistance and radioresistance. Cancer Res 68: 605-614, 2008.

30. Luoto KR, Meng AX, Wasylishen AR, Zhao H, Coackley CL, Penn LZ and Bristow RG: Tumor cell kill by c-MYC depletion: Role of MYC-regulated genes that control DNA double-strand break repair. Cancer Res 70: 8748-8759, 2010.

31. Boeing S, Williamson L, Encheva V, Gori I, Saunders RE, Instrell R, Aygün O, Rodriguez-Martinez M, Weems JC, Kelly GP, et al: Multiomic analysis of the UV-induced DNA damage response. Cell Rep 15: 1597-1610, 2016.

32. Christmann M and Kaina B: Transcriptional regulation of human DNA repair genes following genotoxic stress: Trigger mechanisms, inducible responses and genotoxic adaptation. Nucleic Acids Res 41: 8403-8420, 2013.
33. De Siervi A, De Luca P, Byun JS, Di LJ, Fufa T, Haggerty CM Vazquez E, Moiola C, Longo DL and Gardner K: Transcriptional autoregulation by BRCA1. Cancer Res 70: 532-542, 2010.

34. Wang J, Zuo J, Wang M, Ma X, Gao K, Bai X, Wang N, Xie W and Liu H: Pololike kinase 4 promotes tumorigenesis and induces resistance to radiotherapy in glioblastoma. Oncol Rep 41: 2159-2167, 2019.

35. Morozumi Y, Boussouar F, Tan M, Chaikuad A, Jamshidikia M, Colak G, He H, Nie L, Petosa C, de Dieuleveult M, et al: Atad2 is a generalist facilitator of chromatin dynamics in embryonic stem cells. J Mol Cell Biol 8: 349-362, 2016.

36. Zou JX, Duan Z, Wang J, Sokolov A, Xu J, Chen CZ, Li JJ and Chen HW: Kinesin family deregulation coordinated by bromodomain protein ANCCA and histone methyltransferase MLL for breast cancer cell growth, survival, and tamoxifen resistance. Mol Cancer Res 12: 539-549, 2014. 